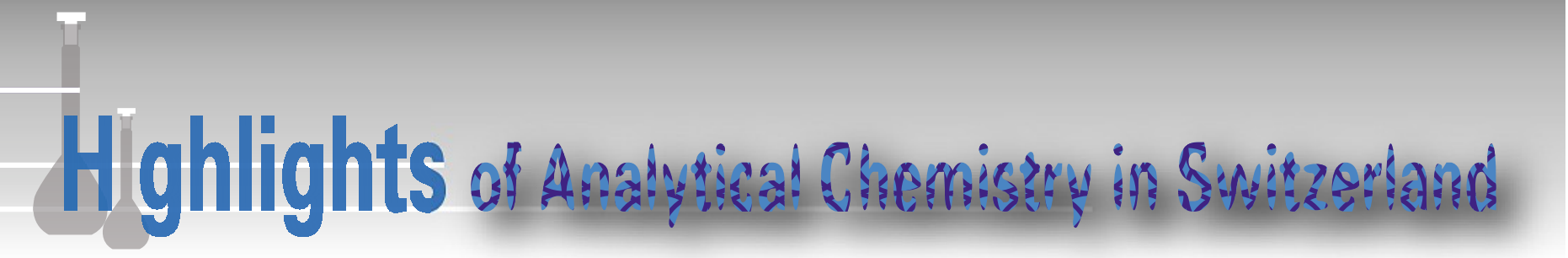

\section{Automated Target Preparation for Gene Expression: Oligonucleotide Microarrays}

\section{Frédéric Raymond*, Sylviane Metairon, and Martin Kussmann}

${ }^{*}$ Correspondence: F. Raymond, Nestlé Research Center, Vers-chez-les-Blanc, CH1000 Lausanne 26

Tel.: +41 2178586 11, Fax: +41 2178594 86, E-Mail: frederic.raymond@rdls.nestle.com

Keywords: Automation - Gene expression analysis .

Microarray target preparation $\cdot$ Standardization

DNA microarray technology allows for massively paralleled monitoring of gene expression, as well as the study of gene regulation and gene interactions at a global scale. Despite a ten-year history of DNA microarray analysis, the technology still suffers from limitations such as inter-experiment variability. While technical differences may influence gene expression results, standardized procedures, high-quality microarrays, and appropriate data collection and transformation are able to generate reproducible and comparable results across experimental replicates and even laboratories. This is especially the case if a common platform and a joint set of procedures are used.

Therefore, standardization of sample preparation and inter-study consistency has to be improved. We have equipped our laboratory with a Hamilton Microlab Star robot programmed to fulfill all requirements of the microarray target preparation. The automated method follows in principle the manual procedure regarding enzymatic reactions, but with substantial technical adaptations for the robot and the external devices. Thus, we implemented a complete method, from the total RNA starting material to the final hybridization mix ready for chip application.

We have examined the reproducibility and quality of the automated RNA target preparation. Our data illustrate good RNA quality and high reproducibility of the automatic RNA target preparation. The variations observed for cRNA yields, cRNA quality, and hybridization intensities across automated target preparations are so low that we suggest the implementation of our robotic method and equipment for microarray analyses in general and on a routine basis. Most importantly, we show that the technical inter-experiment variation is less pronounced than with manually prepared samples. The low variability and the high reproducibility result in a standardized and fully integrated protocol for microarray experiments.

Received: March 22, 2007

Reference

F. Raymond, S. Metairon, R. Borner, M. Hofmann, M. Kussmann, Anal. Chem. 2006, 78, 6299 .

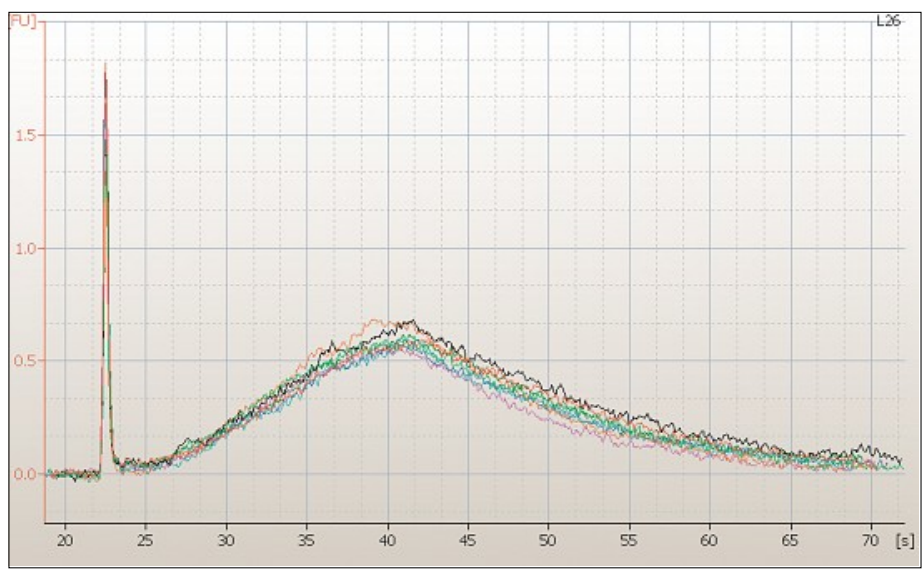

Synthesis of eight cRNA from the same mouse liver total RNA Electropherogram view of Bioanalyser results. The automated target preparation produces cRNA with good quality (peak > $34 \mathrm{sec}$. cRNA fragment size $\approx 1800 \mathrm{bp}$ ).

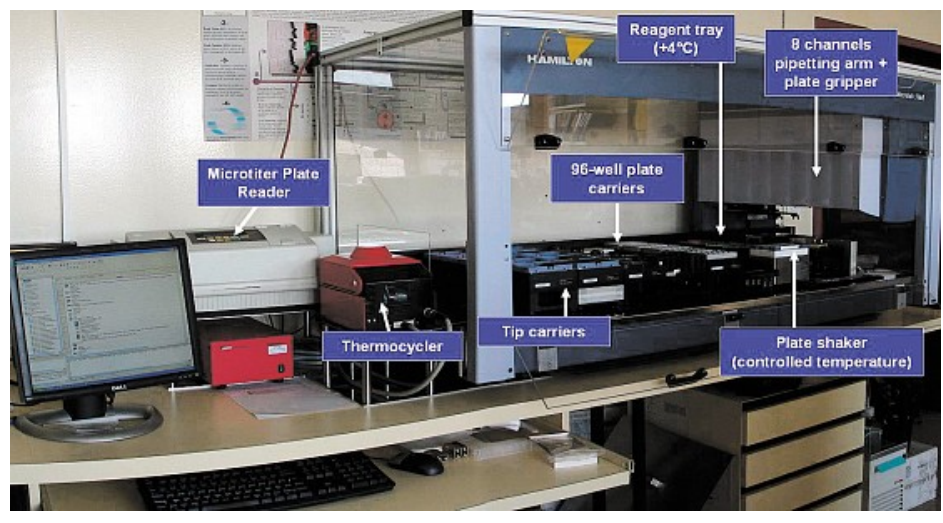

The automation system includes the robotic platform for sample, enzyme, and reaction management, the thermocycler for incubations, and the microtiter plate reader for RNA quantification. The whole set-up is integrated and controlled by the Vector software.

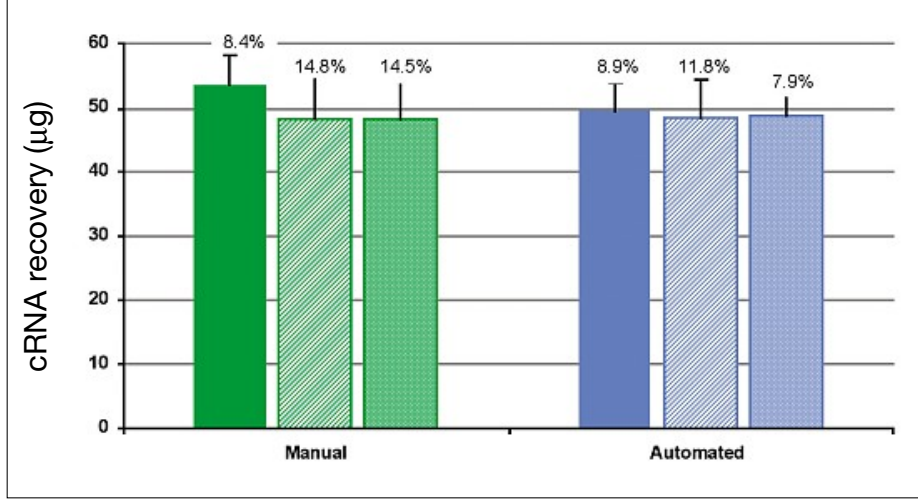

Target preparation. Three series of eight identical mouse RNA samples (5 $\mu \mathrm{g})$ were processed both manually and automatically. While both methods show comparable yields, the automated procedure induces a reduced variability across target preparations. 